\title{
Bioengineered Polymer Nanobeads for Isolation and Electrochemical Detection of Cancer Biomarkers
}

Narshone Soda ${ }^{\mathrm{a}, \mathrm{b}}$, Zennia Jean Gonzaga ${ }^{\mathrm{a}, \mathrm{c}}$, Shuxiong Chen $^{\mathrm{c}}$, Kevin M. Koo ${ }^{\mathrm{e}}$, Nam-Trung

Nguyen $^{\mathrm{b}}$, Muhammad J. A. Shiddiky ${ }^{\mathrm{a}, \mathrm{b}^{*}}$ and Bernd H.A. Rehm ${ }^{\mathrm{c}, \mathrm{d}^{*}}$

${ }^{a}$ School of Environment and Science, Griffith University, Nathan, Queensland 4111, Australia.

${ }^{\mathrm{b}}$ Queensland Micro-and Nanotechnology Centre (QMNC), Griffith University, Nathan, Queensland 4111, Australia.

${ }^{\mathrm{c}}$ Centre for Cell Factories and Biopolymers (CCFB), Griffith Institute for Drug Discovery, Griffith University, Nathan, Queensland 4111, Australia.

${ }^{\mathrm{d}}$ Menzies Health Institute Queensland (MHIQ), Griffith University, Gold Coast, Australia.

${ }^{\mathrm{e}}$ The University of Queensland Centre for Clinical Research (UQCCR), Herston, Queensland 4029, Australia.

* both authors are equal senior authors and corresponding authors. Email:

m.shiddiky@griffith.edu.au; b.rehm@griffith.edu.au

$\$$ both authors contributed equally to this study. 


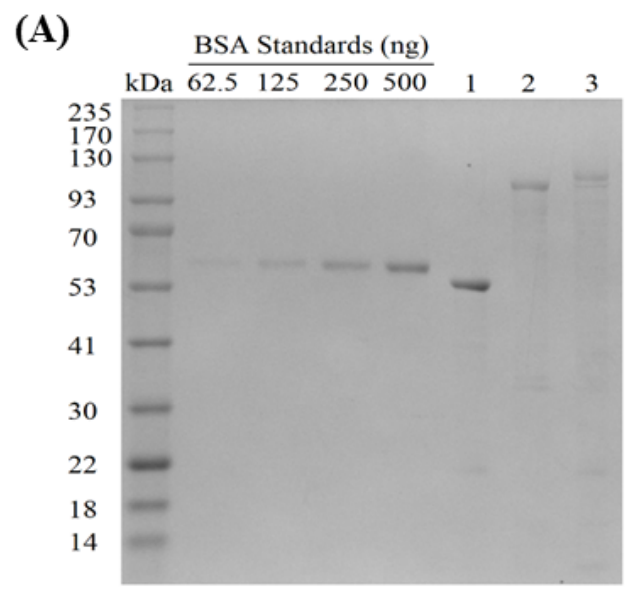

(B)

\begin{tabular}{|l|c|c|c|}
\hline Nanobead Samples & PHB & $\mathbf{Z}_{6}-\mathbf{P H B}$ & $\mathbf{F e}_{\mathbf{3}}-\mathbf{Z}_{\mathbf{6}}-\mathbf{P H B}$ \\
\hline Cultivation volume (L) & 1.50 & 1.50 & 1.50 \\
\hline OD $_{\mathbf{6 0 0}}$ at 0 hr after IPTG induction & 0.54 & 0.52 & 0.48 \\
\hline OD $_{\mathbf{6 0 0}}$ at 48 hr after IPTG induction & 16.80 & 5.60 & 15.60 \\
\hline Wet biomass (g) & 28.80 & 14.10 & 22.00 \\
\hline Wet purified nanobeads (g) & 2.13 & 0.71 & 4.12 \\
\hline Resin or fusion proteins (mg) & 3.71 & 0.79 & 5.05 \\
\hline Resin/biomass (mg/g) & 1.74 & 1.12 & 1.23 \\
\hline
\end{tabular}

Figure S1. Protein concentration and PHB nanobeads yield. (A) Protein concentration measured by densitometry using BSA standards, ranging between 62.5 and $500 \mathrm{ng}$, on 10\% Bis-Tris gel to generate a standard curve. The image was taken by gel doc (BioRad Laboratories, Hercules, CA) and analysed with Image Lab software (BioRad Laboratories, Hercules, CA). Lane 1, PHB (64.2 kDa); lane 2, Z6-PHB (118.3 kDa); lane 3, Fe3-Z6-PHB (119.6 kDa). (B) Table showing PHB nanobeads yield and composition. 

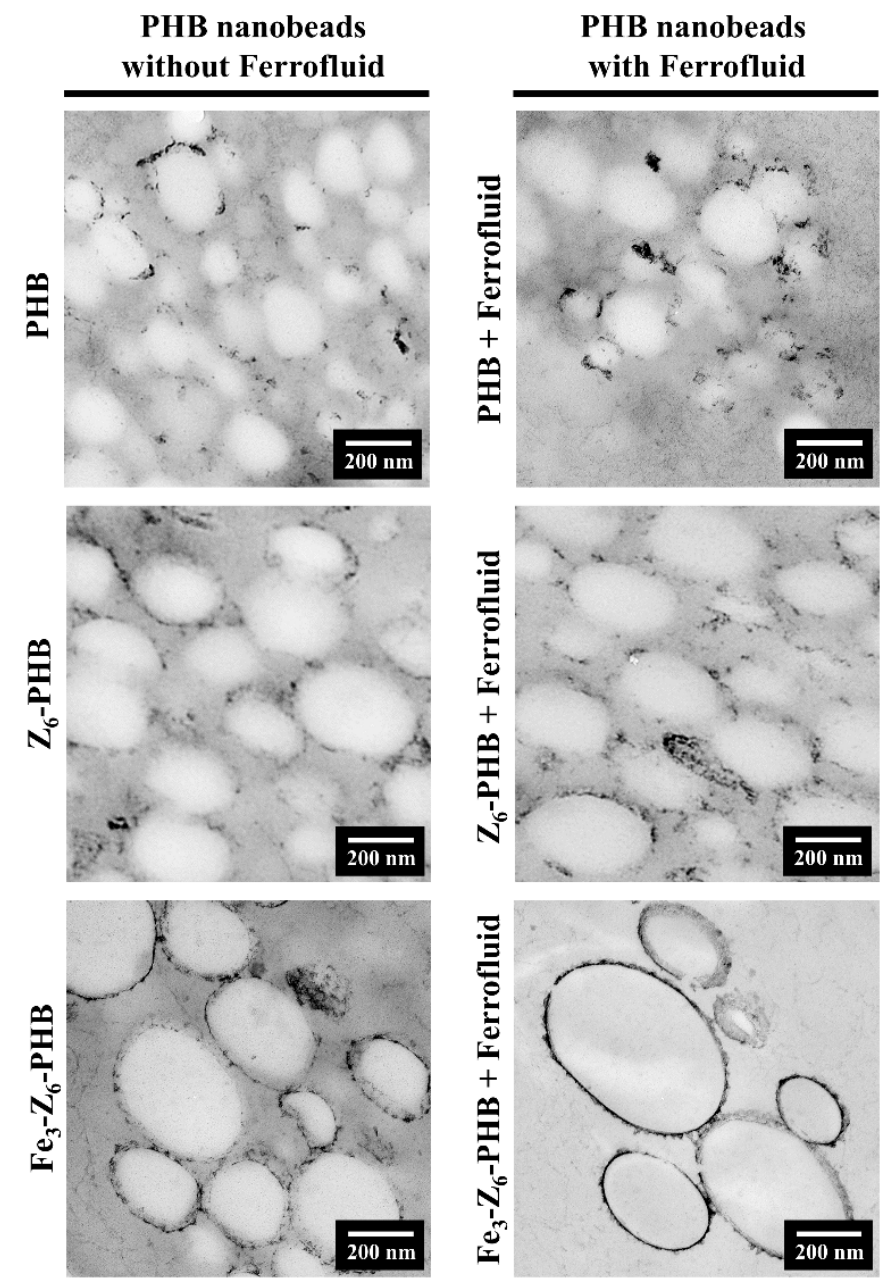

Figure S2. TEM images of various PHB nanobeads. 
(A)

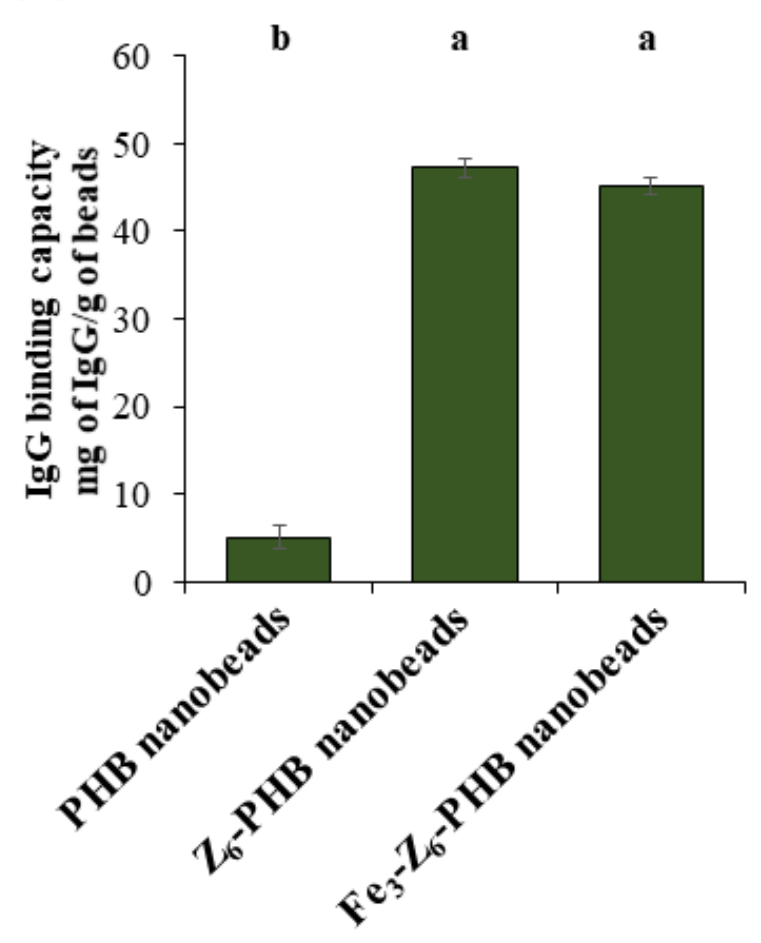

(B)

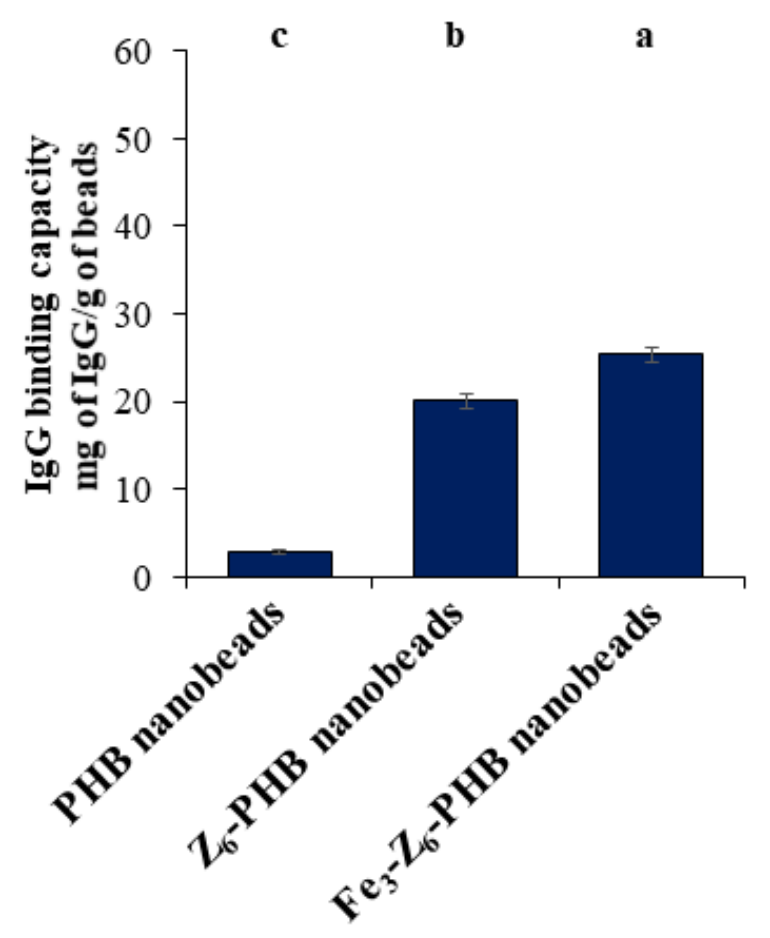

Figure S3. Human IgG-binding capacity of PHB nanobeads displaying ZZ and Fe domains (A) without and (B) with ferrofluid. Protein concentration was measured by Bradford assay using purified human $\operatorname{IgG}$ as the standard; 40-60 mg nanobeads were used for the assay. The mean of IgG-binding capacity is shown \pm the standard error of the mean. All measurements were conducted in triplicates. All PHB nanobeads were produced in recombinant E. coli BL21 (DE3) (pMCS69) harbouring the respective pET14b plasmids. Statistical analysis was done by one-way ANOVA with statistical significance $(\mathrm{p}<0.05)$ indicated by letter-based representation of pairwise comparisons between groups using Tukey's post-hoc test. 
(A)

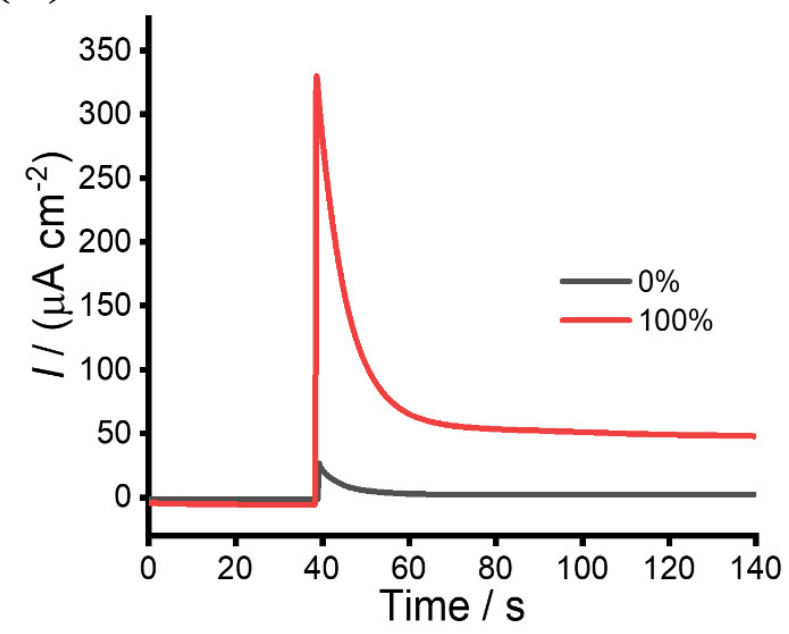

(B)

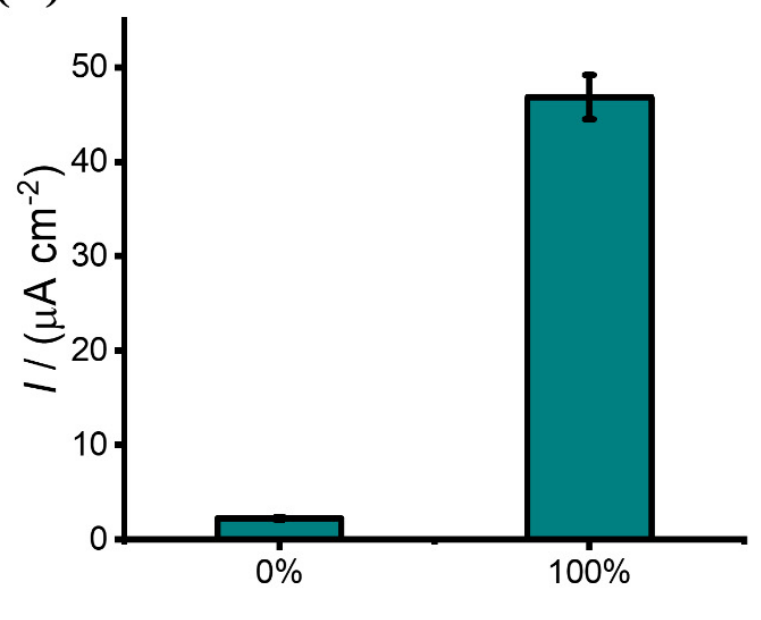

Figure S4. Electrochemical detection of methylated DNA in buffer sequestered by engineered superparamagnetic PHB nanobeads. (A) Current density responses for the detection of DNA methylation in the presence (red) and absence (control) of 100\% methylated DNA targets. (B) Corresponding current densities for the 100\% methylated target (right bar) and blank control (left bar) samples. 
(A)

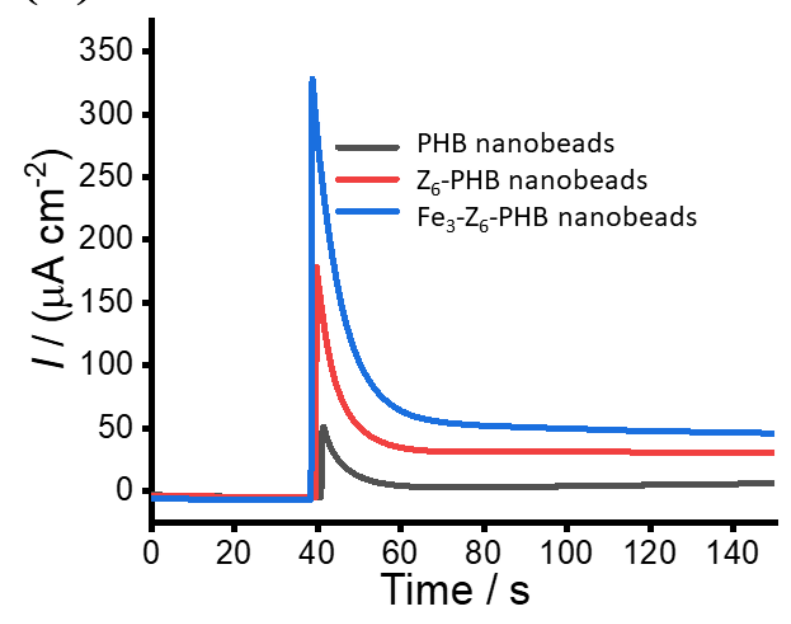

(B)

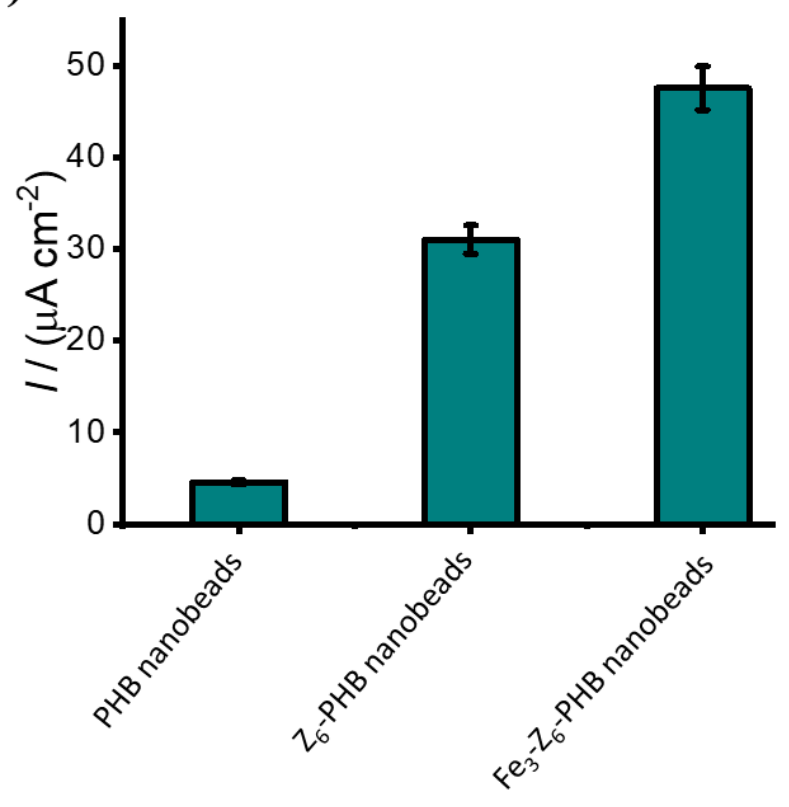

Figure S5. Amperometric response for PHB, $\mathrm{Z}_{6}-\mathrm{PHB}$ and $\mathrm{Fe}_{3}-\mathrm{Z}_{6}-\mathrm{PHB}$ nanobeads to detect $100 \%$ methylated DNA sequences.

Post-functionalization, several experimental conditions were optimized to achieve optimum performance of the electrochemical assay. The selection of PHB nanobeads was determined by measuring the maximum electrochemical responses for methylated DNA targets. Ferrofluid was added to three different types of nanobeads and used to capture, isolate and purify methylated DNA targets. Figure $\mathbf{S} 4$ shows the different current density responses to be $4.57 \mu \mathrm{A} \mathrm{cm}^{-2}$ for $\mathrm{PHB}$, $31.1 \mu \mathrm{A} \mathrm{cm}^{-2}$ for $\mathrm{Z}_{6}-\mathrm{PHB}$ and $47.6 \mu \mathrm{A} \mathrm{cm}^{-2}$ for $\mathrm{Fe}_{3}-\mathrm{Z}_{6}-\mathrm{PHB}$ nanobeads (which is approximately 10 times higher than that of the plain nanobeads). This shows that the $\operatorname{IgG}$ and iron binding domains can improve the assay sensitivity by increasing the target binding efficiency and magnetism. 
Table S1. Description of bacterial strains, plasmids and primers used in this study.

\begin{tabular}{|c|c|c|}
\hline $\begin{array}{lll}\text { Strains, } & \text { Plasmids } & \text { and } \\
\text { Primers } & & \end{array}$ & Relevant characteristics* & References \\
\hline \multicolumn{3}{|l|}{ Bacterial strains } \\
\hline E. coli XL1-Blue & $\begin{array}{l}\text { recAl endA1 gyrA96 thi-1 hsdR17 supE44 } \\
\text { relA1 lac [F' proAB lacI lacZ } \triangle M 15 \text { Tn10 } \\
\left.\left(\text { Tet }^{\mathrm{r}}\right)\right]\end{array}$ & Stratagene \\
\hline E. coli BL21 (DE3) & $\mathrm{F}-o m p T h s d S B(r B-m B-)$ gal dcm (DE3) & Novagen \\
\hline \multicolumn{3}{|l|}{ Plasmids } \\
\hline pET14b & Amp ${ }^{\text {r: }}$ T7 promoter & Novagen \\
\hline pMCS69 & $\begin{array}{l}\mathrm{Cm}^{\mathrm{r}} ; \text { pBBR1MCS derivative encoding } \\
P h a A \text { and } P h a B\end{array}$ & 1 \\
\hline $\begin{array}{l}\text { pET14b_PhaC } \\
\text { (pET14b_PHB) }\end{array}$ & pET-14b derivative encoding $p h a C$ gene & 2 \\
\hline $\begin{array}{l}\text { pET14b_PhaC-Linker-ZZ } \\
\text { (pET14b_PHB-Z2) }\end{array}$ & $\begin{array}{l}\text { pET14b derivative encoding phaC-Linker- } \\
\text { ZZ gene }\end{array}$ & 3 \\
\hline $\begin{array}{l}\text { pET14b_ZZ-Linker-ZZ- } \\
\text { PhaC-Linker-ZZ } \\
\text { (pET14b_Z6-PHB) }\end{array}$ & $\begin{array}{l}\text { pET14b derivative encoding ZZ-Linker- } \\
\text { ZZ-phaC-linker-ZZ gene }\end{array}$ & This study \\
\hline $\begin{array}{l}\text { pET14b_3xFe-ZZ-Linker- } \\
\text { ZZ-PhaC-Linker-ZZ } \\
\text { (pET14b_Fes-Z6-PHB) }\end{array}$ & $\begin{array}{l}\text { pET14b derivative encoding } 3 x F e-Z Z \text { - } \\
\text { Linker-ZZ-phaC-linker-ZZ gene }\end{array}$ & This study \\
\hline \multicolumn{3}{|l|}{ Primers } \\
\hline T7 promoter & TAATACGACTCACTATAGGG & $\begin{array}{l}\text { GenScript, } \\
\text { USA }\end{array}$ \\
\hline T7 terminator & GCTAGTTATTGCTCAGCGG & $\begin{array}{l}\text { GenScript, } \\
\text { USA }\end{array}$ \\
\hline
\end{tabular}

* $\mathrm{Tet}^{\mathrm{r}}$, tetracycline resistance. Amp ${ }^{\mathrm{r}}$, ampicillin resistance. $\mathrm{Cm}^{\mathrm{r}}$, chloramphenicol resistance. 
Table S2. MS-Q-TOF analysis of PHB and PHB-fusion proteins.

\begin{tabular}{|c|c|}
\hline Protein Sequence & $\begin{array}{l}\text { Protein sequence } \\
\text { coverage and the } \\
\text { confirmed fragments }\end{array}$ \\
\hline $\begin{array}{l}\text { PHB (MW: } 64.2 \text { kDa) } \\
1 \text { MATGKGAAASTQEGKSQPFKVTPGPFDPATWLEWSRQWQGTEGNGHAAAS } \\
51 \text { GIPGLDALAGVKIAPAQLGDIQQRYMKDFSALWQAMAEGKAEATGPLHDR } \\
101 \text { RFAGDAWRTNLPYRFAAAFYLLNARALTELADAVEADAKTRQRIRFAISQ } \\
151 \underline{\text { WVDAMSPANF LATNPEAQRLLIESGGESLRAGVRNMMEDLTRGKISQTDE }} \\
201 \underline{\text { SAFEVGRNVAVTEGAVVFENEYFQLLQYKPLTDKVHARPLLMVPPCINKY }} \\
251 \text { YILDLQPESSLVRHVVEQGHTVFLVSWRNPDASMAGSTWDDYIEHAAIRA } \\
301 \underline{\text { IEVARDISGQDKINVLGFCVGGTIVSTALAVLAARGEHPAASVTLLTTLL }} \\
351 \text { DFADTGILDVFVDEGHVQLREATLGGGAGAPCALLRGLELANTFSFLRPN } \\
401 \text { DLVWNYVVDNYLKGNTPVPFDLLFWNGDATNLPGPWYCWYLRHTYLQNEL } \\
451 \underline{\text { KVPGKLTVCGVPVDLASIDVPTYIYGSREDHIVPWTAAYASTALLANKLR }} \\
501 \underline{\text { FVLGASGHIAGVINPPAKNKRSHWTNDALPESPQQWLAGAIEHHGSWWPD }} \\
551 \text { WTAWLAGQAGAKRAAPANYGNARYRAIEPAPGRYVKAKA }\end{array}$ & $\begin{array}{l}\text { 80.3\% } \\
\text { A2-L346, L358-G387, L402- } \\
\text { K413, H443-K520, S522-Q535, } \\
\text { R563-Y584 }\end{array}$ \\
\hline 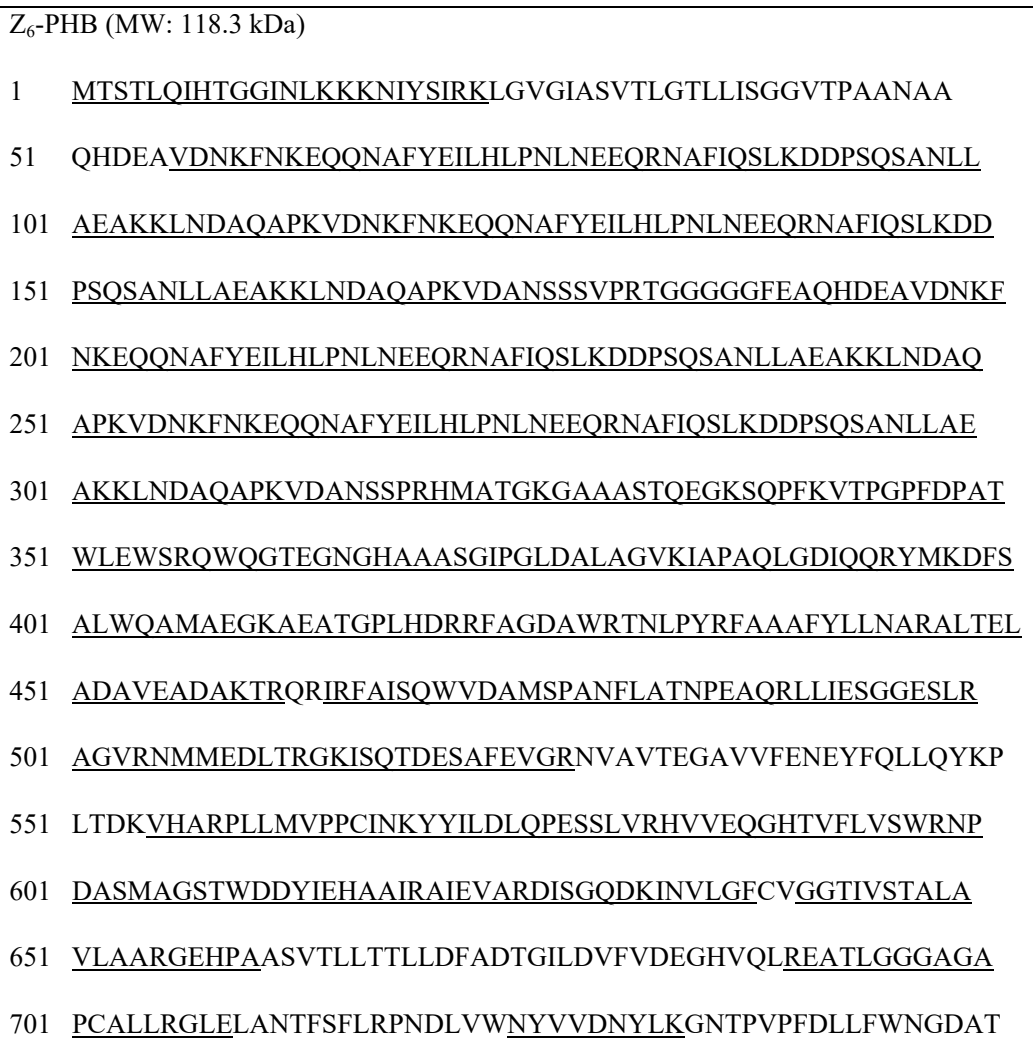 & $\begin{array}{l}82.8 \% \\
\text { M1-K24, V56-R461, I464-R527, } \\
\text { V555-F638, G641-A660, R690- } \\
\text { E709, N725-K733, H763-K840, } \\
\text { A876-Q1079 }\end{array}$ \\
\hline
\end{tabular}




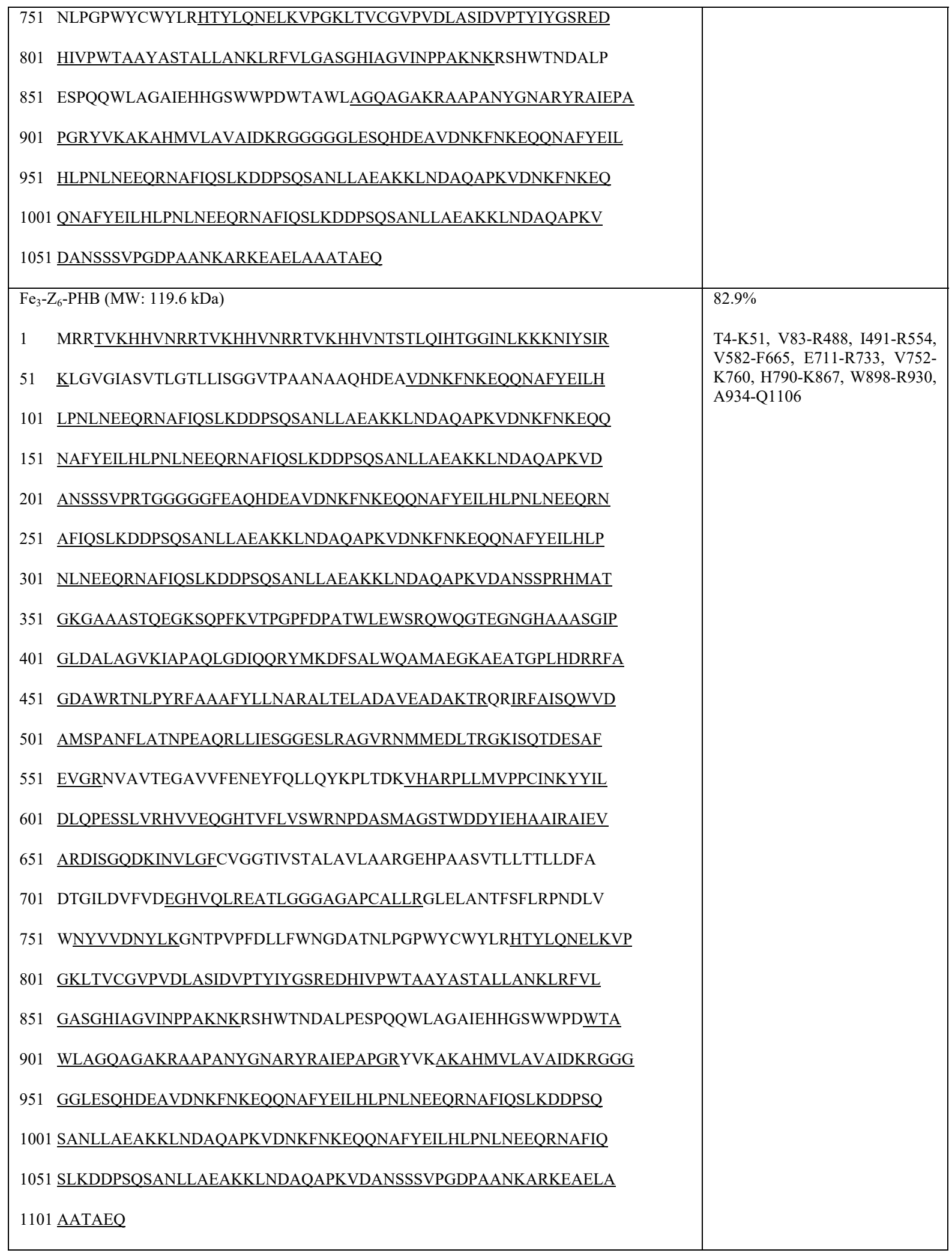

*Confirmed sequences are underlined. MW (Molecular weight). 
Table S3. Particle size and zeta-potential measurements.

\begin{tabular}{|l|l|l|l|l|l|}
\hline PHB nanobeads & $\begin{array}{l}\text { Ave. } \\
\text { Size } \\
(\mathbf{n m})\end{array}$ & $\begin{array}{l}\text { Std. } \\
\text { dev. } \\
\text { (Size) }\end{array}$ & $\begin{array}{l}\text { Ave. } \\
\text { PDI }\end{array}$ & $\begin{array}{l}\text { Ave. Zeta } \\
\text { Potential (mV) }\end{array}$ & $\begin{array}{l}\text { Std. dev. (Zeta } \\
\text { Potential) }\end{array}$ \\
\hline PHB nanobeads & 151 & 8.31 & 0.38 & -15.77 & 0.64 \\
\hline$Z_{6}$-PHB nanobeads & 246 & 32.47 & 0.34 & -20.03 & 0.25 \\
\hline $\begin{array}{l}\text { Fe3-Z6-PHB nanobeads } \\
\text { PHB nanobeads + }\end{array}$ & 424 & 24.92 & 0.29 & -15.17 & 0.47 \\
\hline Ferrofluid & 7.81 & 0.67 & -9.03 & 0.49 \\
\hline $\begin{array}{l}\text { Z6-PHB nanobeads + } \\
\text { Ferrofluid }\end{array}$ & 469 & 43.64 & 0.49 & -12.43 & 1.44 \\
\hline $\begin{array}{l}\text { Fe3-Z6-PHB nanobeads } \\
+ \text { Ferrofluid }\end{array}$ & 504 & 47.86 & 0.38 & 2.53 & 0.90 \\
\hline
\end{tabular}

Ave. (Average); Std. dev. (Standard deviation); PDI (Polydispersity index) 


\section{REFERENCES}

(1) Amara, A. A.; Rehm, B. H. Replacement of the catalytic nucleophile cysteine-296 by serine in class II polyhydroxyalkanoate synthase from Pseudomonas aeruginosa-mediated synthesis of a new polyester: identification of catalytic residues. Biochemical Journal 2003, 374 (2), 413-421.

(2) Peters, V.; Rehm, B. H. A. In vivo monitoring of PHA granule formation using GFP-labeled PHA synthases. FEMS Microbiol. Lett. 2005, 248 (1), 93-100.

(3) Jahns, A. C.; Maspolim, Y.; Chen, S.; Guthrie, J. M.; Blackwell, L. F.; Rehm, B. H. In vivo self-assembly of fluorescent protein microparticles displaying specific binding domains. Bioconjugate chemistry 2013, 24 (8), 1314-1323. 